\title{
Alvimopan for the Management of Postoperative Ileus After Bowel Resection: Characterization of Clinical Benefit by Pooled Responder Analysis
}

\author{
Kirk Ludwig • Eugene R. Viscusi • Bruce G. Wolff • \\ Conor P. Delaney $\cdot$ Anthony Senagore $\cdot$ Lee Techner
}

Published online: 5 June 2010

(C) Société Internationale de Chirurgie 2010

\begin{abstract}
Background A pooled post hoc responder analysis was performed to assess the clinical benefit of alvimopan, a peripherally acting mu-opioid receptor (PAM-OR) antagonist, for the management of postoperative ileus after bowel resection.

Methods Adult patients who underwent laparotomy for bowel resection scheduled for opioid-based intravenous patient-controlled analgesia received oral alvimopan or placebo preoperatively and twice daily postoperatively until hospital discharge or for 7 postoperative days. The
\end{abstract}

Clinical Trials Registration Numbers NCT00388258, NCT00388479, NCT00388401, NCT00205842

K. Ludwig $(\square)$

Department of Surgery, Medical College of Wisconsin 9200 West Wisconsin Avenue, Milwaukee, WI 53226, USA

e-mail: kludwig@mcw.edu

E. R. Viscusi

Department of Anesthesiology, Thomas Jefferson University,

111 S 11th Street, Suite G-8490, Philadelphia, PA 19107, USA

B. G. Wolff

Division of Colon and Rectal Surgery, Mayo Clinic, 200 First

Street, S.W., Gonda 9-205 CRS, Rochester, MN 55905, USA

C. P. Delaney

Division of Colorectal Surgery, University Hospitals of Cleveland, Case Western Reserve University, 11100 Euclid Avenue, Cleveland, OH 44106, USA

A. Senagore

Department of Surgery, Spectrum Health System, 100 Michigan

NE, MC005, Grand Rapids, MI 49503, USA

L. Techner

Adolor Corporation, Exton Office, 700 Pennsylvania Drive,

Exton, PA 19341, USA proportion of responders and numbers needed to treat (NNT) were examined on postoperative days (POD) 3-8 for GI-2 recovery (first bowel movement, toleration of solid food) and hospital discharge order (DCO) written.

Results Alvimopan significantly increased the proportion of patients with GI-2 recovery and DCO written by each POD $(P<0.001$ for all). More patients who received alvimopan achieved GI-2 recovery on or before POD 5 (alvimopan, 80\%; placebo, 66\%) and DCO written before POD 7 (alvimopan, 87\%; placebo, 72\%), with corresponding NNTs equal to 7 .

Conclusions On each POD analyzed, alvimopan significantly increased the proportion of patients who achieved GI-2 recovery and DCO written versus placebo and was associated with relatively low NNTs. The results of these analyses provide additional characterization and support for the overall clinical benefit of alvimopan in patients undergoing bowel resection.

\section{Introduction}

Postoperative ileus (POI) is an important clinical problem that occurs after major abdominal operations and is characterized by the inability to tolerate solid food, absence of passage of flatus and stool, pain and abdominal distension, nausea, vomiting, lack of bowel sounds, and accumulation of gas and fluids in the bowel [1]. Both endogenous opioids released in the gastrointestinal (GI) tract in response to stress and exogenous opioids used for pain management contribute to the complex etiology of POI $[2,3]$.

Postoperative ileus is associated with prolonged hospital length of stay (LOS), readmission, and increased risk for postoperative morbidity [4-8]. Gastrointestinal recovery is generally expected within 5 days (early recovery period) of 
bowel resection (BR) [9] and recovery delayed beyond 5 postoperative days (PODs) of BR (late recovery period) increases patient risk for morbidity and the probability of extending LOS [4, 5, 10-12]. Based on the placebo arms of alvimopan trials (mean discharge order [DCO] written = 6.1 days) [13] and Health Care Financing Administration database of major intestinal resections in 150 U.S. hospitals (mean LOS $=6.5$ days) [14], a LOS of 7 days or more may be considered prolonged. Furthermore, national LOS statistics (including data representing more than 340,000 U.S. discharges in 1,054 U.S. hospitals) for large and small BR indicate that average LOS after these operations is substantially higher: 10 to 15 days [15]. Prolonged LOS may be associated with increased postoperative morbidity, such as nosocomial infections [16]. In addition to the clinical burden of POI, according to an analysis of a national database, hospitalization costs for patients with coded POI were substantially higher compared with patients without coded POI [10]. Furthermore, there is only one FDAapproved pharmacologic agent for the acceleration of GI recovery after BR.

Alvimopan (Entereg ${ }^{\circledR}$, Adolor Corporation, Exton, PA), a recently approved peripherally acting mu-opioid receptor (PAM-OR) antagonist, was designed to mitigate the peripheral GI-related adverse effects of opioids without compromising centrally based analgesia [17]. Alvimopan was well tolerated, accelerated GI recovery, and reduced the time to hospital DCO written and POI-related morbidity after BR without compromising opioid-based analgesia in phase III efficacy trials [4, 18-22]. Although important, these components alone do not provide a complete assessment of the clinical benefit of a new therapy for the management of POI.

Therefore, a responder analysis, which takes individual responses to treatment into account, was performed to investigate further the clinically meaningful benefit of alvimopan for the management of POI after BR. This analysis investigated GI recovery and hospital DCO written over time during the early (PODs 3-5) and late (PODs 6-8) recovery periods in patients who received alvimopan or placebo in North American phase III efficacy trials [18-22].

\section{Patients and methods}

Adult patients (age $\geq 18$ years) undergoing laparotomy for partial small or large BR with primary anastomosis and who were scheduled for postoperative pain management with intravenous opioid-based patient-controlled analgesia were eligible for enrollment [18-22]. Patients were excluded from eligibility if they were pregnant, currently using opioids or received an acute course of opioids
(>3 doses) within 1 week of study entry, had a complete bowel obstruction, were undergoing total colectomy, colostomy, ileostomy, or coloanal or ileal pouch-anal anastomosis, or had a history of total colectomy, gastrectomy, gastric bypass, short bowel syndrome, or multiple previous abdominal operations performed by laparotomy. All patients signed a written, informed consent that was approved by individual institutional review boards [18-22].

Study design and treatments

This was a pooled post hoc analysis of four randomized, double-blind, placebo-controlled, phase III trials (14CL302, 14CL308, 14CL313, and 14CL314) [18-22]. The majority $(>90 \%)$ of patients analyzed received placebo or alvimopan $12 \mathrm{mg}$ at least 30 minutes but no longer than 5 hours before surgery and then twice daily after surgery until hospital discharge or for a maximum of 7 PODs (15 doses) while hospitalized. A multimodal, standardized accelerated postoperative care pathway was implemented in each study to facilitate GI recovery consistent with best-care practices: if the nasogastric tube (NGT) was kept in place after surgery, it was removed no later than noon on POD 1 before the first postoperative dose of study medication was administered; a liquid diet was offered and ambulation was encouraged on POD 1; solid food was offered on POD 2 [14].

\section{Assessments}

Gastrointestinal recovery was assessed by a composite measurement (GI-2), which included recovery of upper (toleration of solid food) and lower (first bowel movement) GI function, with time to achieve GI-2 based on the last event to occur. Postoperative LOS was defined as the calendar day of surgery to the calendar day of DCO written.

Responder analyses were performed at six cutoff time points on PODs 3 through 8. Postsurgery days (PSDs) were defined as 24-hour intervals after the end of surgery time (last suture or staple). Patients were considered responders if they achieved GI-2 recovery or DCO written by the cutoff time point and did not experience subsequent complications of POI. Complications of POI included prolonged LOS or readmission within 7 days after initial hospital discharge attributable to POI, paralytic ileus, or small intestinal obstruction reported as serious adverse events. Number needed-to-treat (NNT) analyses were performed to provide an estimate of the number of patients who would need to be treated to attain a favorable outcome. This type of analysis is directly applicable to clinical practice because it demonstrates the effort required to achieve a particular therapeutic target (e.g., achieving GI recovery within 5 days or discharge from the hospital less than 1 week from surgery) $[22,23]$ and is calculated from 
the absolute difference in the proportion of patients in alvimopan- and placebo-treated groups achieving an event.

Statistical methods

Fisher's exact test was used to evaluate the treatment effect on the proportion of responders at each cutoff time point. All statistical analyses were performed using $\mathrm{SAS}^{\circledR}$ version 9.1 or higher (SAS Institute, Cary, NC).

\section{Results}

Patients

Of the 2,281 patients randomized in the 4 phase III trials, 1,409 patients underwent BR, received placebo or alvimopan, and were included in the modified intent-to-treat population (Table 1) [4]. Approximately half of all patients were women (51\%), and the mean age was 61 years. The most common reason for surgery was colon or rectal cancer $(51 \%)$.

\section{Assessments}

Overall, alvimopan significantly increased the proportion of patients who achieved GI-2 recovery on each day of the early and late recovery periods $(P<0.001$ for all; Fig. 1a). Eighty percent of patients in the alvimopan group compared with $66 \%$ of patients in the placebo group achieved GI-2 recovery on or before POD 5. Moreover, alvimopan significantly increased the proportion of patients who achieved GI-2 recovery on each day of the early and late recovery periods irrespective of age or sex ( $P \leq 0.03$ for all). Significant increases $(P<0.001$ for all $)$ in the proportion of patients who achieved GI-2 recovery on each day were observed in white patients; however, significant increases $(P<0.05)$ were not observed in non-white patients on PSD 3 and 5. Only seven patients would need to be treated with alvimopan to reduce the risk associated with longer GI recovery (GI-2 recovery $>$ 5 PSD) for one patient (Fig. 1b).

Alvimopan also significantly increased the proportion of patients who received DCO written on each day of the early and late recovery periods ( $P \leq 0.001$ for all; Fig. 2a). Consistent with GI-2 recovery results, more patients in the alvimopan group (87\%) received DCO written before POD 7 compared with patients in the placebo group (72\%). Alvimopan significantly increased the proportion of patients who received DCO written on each day of the early and late recovery periods regardless of age $(P \leq$ 0.025 for all). Significant increases $(P<0.001$ for all) were observed regardless of sex on each day except for PSD 3 in women. Significant increases $(P<0.001$ for all $)$ in the
Table 1 Patient demographics and baseline surgery characteristics (modified intent-to-treat population) $^{\mathrm{a}}$

\begin{tabular}{|c|c|c|}
\hline & $\begin{array}{l}\text { Placebo } \\
(n=695)\end{array}$ & $\begin{array}{l}\text { Alvimopan } \\
(n=714)\end{array}$ \\
\hline \multicolumn{3}{|l|}{ Age (years) } \\
\hline Mean $\pm \mathrm{SD}$ & $60.4 \pm 14.1$ & $60.7 \pm 14.6$ \\
\hline Patients $\geq 65$ & $291(41.9)$ & $308(43.1)$ \\
\hline \multicolumn{3}{|l|}{ Race } \\
\hline White & $589(84.7)$ & $599(83.9)$ \\
\hline Black & $67(9.6)$ & $77(10.8)$ \\
\hline Hispanic & $28(4.0)$ & $29(4.1)$ \\
\hline Asian & $8(1.2)$ & $7(1.0)$ \\
\hline Native American & $1(0.1)$ & $1(0.1)$ \\
\hline Other & $2(0.3)$ & $1(0.1)$ \\
\hline \multicolumn{3}{|l|}{ Sex } \\
\hline Female & $362(52.1)$ & $358(50.1)$ \\
\hline \multicolumn{3}{|l|}{$B M I$} \\
\hline Mean $\pm \mathrm{SD}\left(\mathrm{kg} / \mathrm{m}^{2}\right)$ & $28.5 \pm 6.2$ & $27.7 \pm 6$ \\
\hline Patients with $\mathrm{BMI} \geq 30 \mathrm{~kg} / \mathrm{m}^{2}$ & $231(33.2)$ & $203(28.4)$ \\
\hline \multicolumn{3}{|l|}{ Primary reason for surgery } \\
\hline Colon or rectal cancer & $349(50.2)$ & $374(52.4)$ \\
\hline Diverticular disease & $114(16.4)$ & $109(15.3)$ \\
\hline Takedown & $62(8.9)$ & $73(10.2)$ \\
\hline Intestinal polyps & $65(9.4)$ & $56(7.8)$ \\
\hline Crohn's disease & $35(5.0)$ & $49(6.9)$ \\
\hline Other & $70(10.1)$ & $53(7.4)$ \\
\hline \multicolumn{3}{|l|}{ Surgery type } \\
\hline Small BR & $50(7.2)$ & $65(9.1)$ \\
\hline Large BR & $645(92.8)$ & $649(90.9)$ \\
\hline \multicolumn{3}{|l|}{ Surgery duration $(h)$} \\
\hline Overall mean $\pm \mathrm{SD}$ & $2.2 \pm 1.1$ & $2.1 \pm 1.1$ \\
\hline
\end{tabular}

There were no significant differences in patient demographics or baseline characteristics between treatment groups. Data are numbers with percentages in parentheses unless otherwise indicated. Adapted with permission from Elsevier: Wolff BG, Weese JL, Ludwig KA et al (2007) Postoperative ileus-related morbidity profile in patients treated with alvimopan after bowel resection. J Am Coll Surg 204:609-616

$S D$ standard deviation; $B M I$ body mass index; $B R$ bowel resection

proportion of patients who received DCO written on each day were observed in white patients. Significant increases $(P<0.05)$ were observed for non-white patients on each day except PSD 3. The proportion of patients who remained in the hospital on or after PSD 7 was reduced from $34 \%$ in the placebo group to $19 \%$ in the alvimopan group $(P<0.001$; NNT $=7)$. Similarly, the proportion of elderly patients ( $\geq 65$ years old) who received DCO written on or after PSD 7 was reduced from $35 \%$ in the placebo group to $17 \%$ in the alvimopan group $(\mathrm{NNT}=5)$. Overall, NNT analysis indicated that seven patients would need to receive alvimopan to reduce the risk associated with 

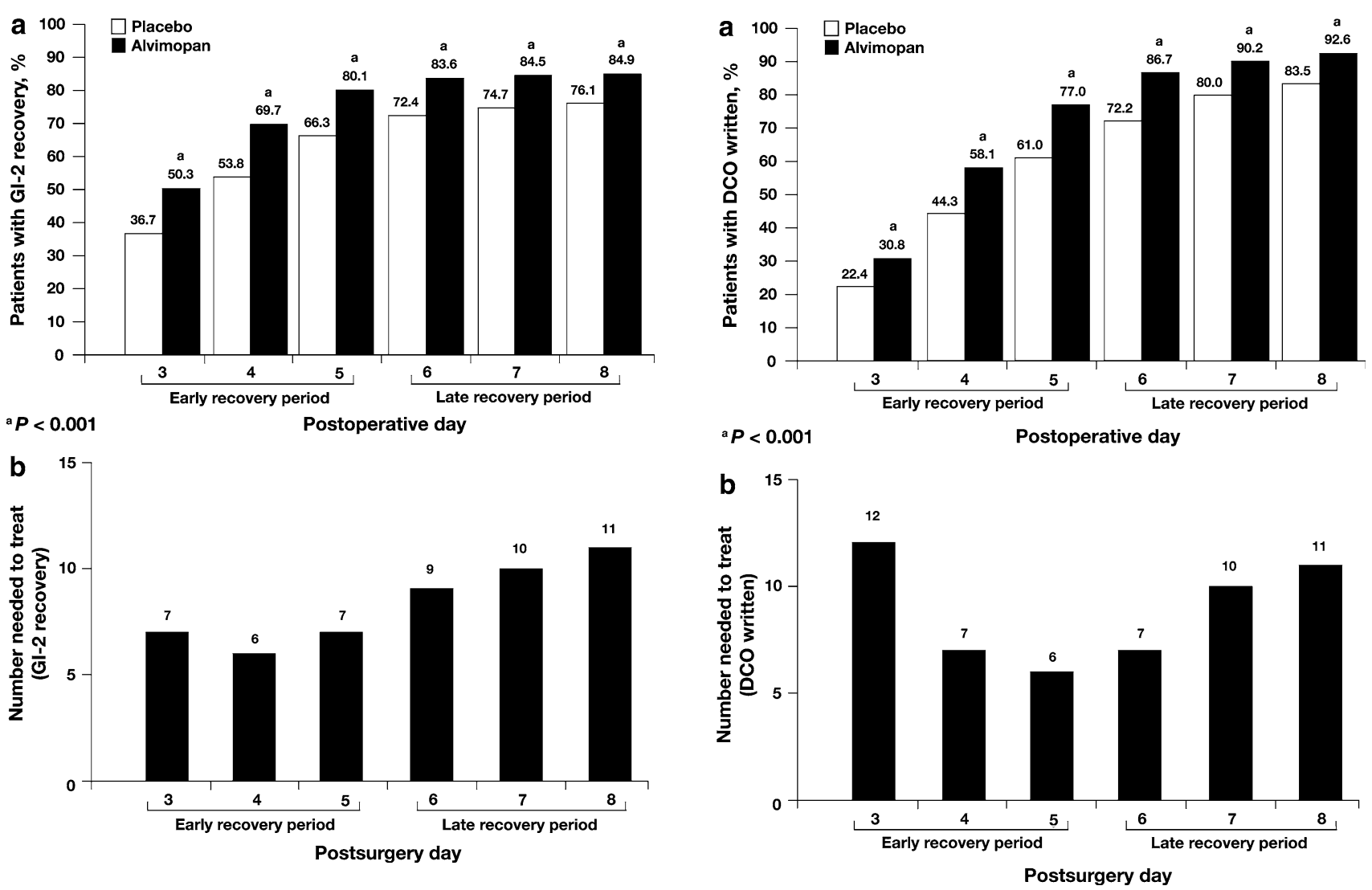

Fig. 1 Proportion of patients achieving GI-2 recovery by calendar day (a) and the number needed to treat to prevent delayed GI-2 recovery by postsurgery day (b) (modified intent-to-treat population). GI-2 time to first toleration of solid food and first bowel movement

Fig. 2 Proportion of patients receiving discharge order written by calendar day (a) and the number needed to treat to prevent delayed discharge order written by postsurgery day (b) (modified intent-totreat population)

prolonged LOS (DCO written $\geq 7$ PSD) for one patient (Fig. 2b). Postoperative LOS was 1 day shorter in the alvimopan group (5.6 days) compared with the placebo group ( 6.6 days; $P<0.001$ ).

The rates of readmission for any cause within 10 days (placebo group, $8 \%$; alvimopan group, $5 \% ; P=0.01$; NNT $=33$ ) and readmission resulting from complications of POI within 7 days (placebo group, 2\%; alvimopan group, $1 \% ; P$ $=0.126$; NNT $=100$ ) were low in both groups. Of those patients who recovered GI-2 function in the early recovery period (placebo group, $n=461$; alvimopan group, $n=572$ ), $7 \%$ of patients in the placebo group and $4 \%$ of patients in the alvimopan group were readmitted to the hospital for any cause $(P=0.043)$, and no patient in either group was readmitted for POI, paralytic ileus, or small bowel obstruction. Furthermore, $12 \%$ of patients in the placebo group $(n=234)$ and $10 \%$ of patients in the alvimopan group $(n=142)$ who recovered GI-2 function in the late recovery period were readmitted for any cause $(P=0.614)$, and $6 \%$ of patients in the placebo group and $5 \%$ of patients in the alvimopan group were readmitted for POI, paralytic ileus, or small bowel obstruction $(P=0.818)$.

\section{Discussion}

Although there are no validated patient-reported outcome measures for POI, there is general agreement that providing a reduction in the time to GI recovery after BR is clinically meaningful to the patient. In addition to clinical benefit, it was previously reported that hospitalization costs for patients with an International Classification of Diseases, ninth revision (ICD-9), coded POI were substantially higher than for patients without coded POI $(\$ 18,877$ vs. $\$ 9,460)$ and resulted in longer hospital LOS (11.5 vs. 5.5 days) [10]. The projected annual costs attributed to managing coded POI is $\$ 1.46$ billion; however, this was estimated based on discharge coding of POI and likely underestimates the true prevalence rate [10]. Thus, in addition to the clinical burden associated with POI, a substantial economic burden is apparent.

Previously reported primary efficacy and morbidity analyses of alvimopan trials have demonstrated that alvimopan can significantly accelerate GI recovery in conjunction with a standardized accelerated postoperative care pathway without compromising opioid-based analgesia or 
increasing POI-related morbidity and was generally well tolerated [4, 13, 18-22]. Patients in the alvimopan-treated groups recovered both upper and lower GI function earlier compared with patients in the placebo-treated groups [13, 18-22].

The current responder analysis confirmed that alvimopan significantly increased the proportion of patients who achieved GI-2 recovery during both the early and late recovery periods in patients who underwent BR via laparotomy. Alvimopan also significantly increased the proportion of patients who received DCO written during both the early and late recovery periods. In general, GI-2 and DCO written were accelerated with alvimopan use regardless of age or sex; results for race were inconsistent, most likely because of the smaller sample size for nonwhite patients $(n=221)$ compared with white patients $(n=$ 1,188). Patients treated with alvimopan were discharged from the hospital 1 day earlier than patients who received placebo, and rates of readmission were lower in the alvimopan group compared with the placebo group. Moreover, NNT analysis revealed that only a small number of patients $(\mathrm{NNT}=7)$ would need to be treated to achieve risk reduction for one patient experiencing delayed GI recovery or prolonged LOS. In addition, the numbers needed to treat to reduce the risk of prolonged LOS in elderly patients was further reduced $(\mathrm{NNT}=5)$. Hence, alvimopan use was associated with shifting more patients into the earlier phase of the recovery process. Moreover, NNT calculations indicated that 33 patients would need to be treated to prevent one readmission for any cause. A limitation of this NNT analysis is that by grouping patients together, it is assumed that patients who achieved or did not achieve GI-2 recovery on each day had an equal level of response, which is not the case. Thus, this analysis may underestimate the benefit of alvimopan.

By comparison, preventive therapies, such as the use of prophylactic antibiotic regimens to prevent postoperative wound infections in patients undergoing colorectal surgery or use of low molecular weight heparin or graduated compression stockings to prevent deep vein thrombosis, have NNTs ranging from 4 to 17 (calculated using absolute risk reduction) and have been incorporated into standard clinical practice based on benefits demonstrated in clinical trials [23-26] Indeed, wound infection after colorectal surgery was associated with an increase of approximately 12 hospital days and \$1,500-\$8,400 in costs, highlighting the value of instituting preventive therapies such as prophylactic antibiotic use into clinical practice [25]. By comparison, POI after colorectal surgery was associated with an increase of 4.9 hospital days and \$8,296 in costs [27]. The NNTs for prevention therapies, such as prophylactic antibiotic use before colorectal surgery, provide support that a therapy (e.g., alvimopan) for an acute postoperative condition (e.g., POI) with similar NNTs (without increased risk) may represent meaningful patient benefit if incorporated into standard clinical practice, particularly because POI is recognized as the most common cause for increased LOS in these patients, and increased LOS after major surgery often is associated with increased risk for nosocomial complications [11, 28]. Additionally, the overall rate of POI is generally underreported and/or underrecognized, suggesting that the overall burden of POI on the health care system may be substantially underestimated [27].

Elderly patients (older than aged 60 years) are at a higher risk for postoperative morbidity and prolonged LOS after colorectal surgeries compared with younger patients [14, 29-32]. In this responder analysis, alvimopan significantly increased the proportion of patients who received DCO written during the early and late recovery periods and significantly reduced the proportion of patients with prolonged hospital stays (DCO written after 7 PODs) in both the overall and elderly trial populations compared with placebo. Therefore, these data indicate that alvimopan may be beneficial to patients who are at greater risk for prolonged LOS, such as the elderly. Such reductions in hospital LOS, prolonged LOS, and complications of POI (e.g., NGT insertion) may provide a benefit to patients and the healthcare system $[5,6]$.

In phase III trials, the accelerated GI recovery observed in patients who received alvimopan was not associated with additional complications of POI [4, 18-22]. Postoperative ileus-related morbidities, such as postoperative NGT insertion and prolonged LOS, were lower in patients who received alvimopan compared with placebo [4]. Furthermore, alvimopan was well tolerated in all phase III trials [18-22]. In a recent pooled analysis of patients who underwent $\mathrm{BR}$ in three phase III efficacy trials, the most common treatment-emergent adverse events were nausea and vomiting, and the incidences of these were lower in the alvimopan group compared with the placebo group $(P<$ 0.05) [13].

Collectively, the data from this responder analysis in conjunction with previously reported efficacy, morbidity, and safety data provide a more complete assessment of the clinical benefit of alvimopan for the management of POI in patients who undergo BR. Alvimopan, therefore, may provide earlier GI recovery and discharge from the hospital without increased morbidity or interference with opioid pain management to a greater proportion of patients than what may be provided by a standardized accelerated postoperative care pathway alone. 
Acknowledgments Support for studies described in this report and funding for medical editorial assistance was provided by Adolor Corporation, Exton, Pennsylvania, and GlaxoSmithKline, Philadelphia, PA. The authors thank Kerry O. Grimberg, PhD, and Bret A. Wing, PhD, ProEd Communications, Inc., for their medical editorial assistance with this manuscript.

Conflict of Interest Disclosures Dr. Ludwig has nothing to disclose; Dr. Viscusi has received grant support from and is a consultant for Adolor Corporation; Dr. Wolff has been on the advisory board and has consulted for Adolor Corporation; Dr. Delaney is on the advisory board and is a consultant for Adolor Corporation; Dr. Senagore is on the advisory board and is a consultant for Adolor Corporation; Dr. Techner is an employee of Adolor Corporation.

\section{References}

1. Behm B, Stollman N (2003) Postoperative ileus: etiologies and interventions. Clin Gastroenterol Hepatol 1:71-80

2. Kehlet H, Holte K (2001) Review of postoperative ileus. Am J Surg 182(Suppl):3S-10S

3. Lundgren O (2000) Sympathetic input into the enteric nervous system. Gut 47(Suppl 4):iv33-iv35; discussion iv36

4. Wolff BG, Weese JL, Ludwig KA et al (2007) Postoperative ileus-related morbidity profile in patients treated with alvimopan after bowel resection. J Am Coll Surg 204:609-616

5. Oderda GM, Said Q, Evans RS et al (2007) Opioid-related adverse drug events in surgical hospitalizations: impact on costs and length of stay. Ann Pharmacother 41:400-406

6. Kariv Y, Wang W, Senagore AJ et al (2006) Multivariable analysis of factors associated with hospital readmission after intestinal surgery. Am J Surg 191:364-371

7. Chang SS, Baumgartner RG, Wells N et al (2002) Causes of increased hospital stay after radical cystectomy in a clinical pathway setting. J Urol 167:208-211

8. Delaney CP, Senagore AJ, Viscusi ER et al (2006) Postoperative upper and lower gastrointestinal recovery and gastrointestinal morbidity in patients undergoing bowel resection: pooled analysis of placebo data from 3 randomized controlled trials. Am J Surg 191:315-319

9. Delaney CP (2004) Clinical perspective on postoperative ileus and the effect of opiates. Neurogastroenterol Motil 16(Suppl 2):61-66

10. Goldstein JL, Matuszewski KA, Delaney CP et al (2007) Inpatient economic burden of postoperative ileus associated with abdominal surgery in the United States. P\&T 32:82-90

11. Person B, Wexner SD (2006) The management of postoperative ileus. Curr Probl Surg 43:6-65

12. Kehlet H, Buchler MW, Beart RW et al (2006) Care after colonic operation-is it evidence-based? Results from a multinational survey in Europe and the United States. J Am Coll Surg 202:4554

13. Delaney CP, Wolff BG, Viscusi ER et al (2007) Alvimopan, for postoperative ileus following bowel resection. A pooled analysis of phase III studies. Ann Surg 245:355-363

14. Delaney CP, Fazio VW, Senagore AJ et al (2001) "Fast track" postoperative management protocol for patients with high comorbidity undergoing complex abdominal and pelvic colorectal surgery. Br J Surg 88:1533-1538
15. H-CUPnet: national and regional estimates on hospital use for all patients from the HCUP Nationwide Inpatient Sample. http:// hcupnet.ahrq.gov/HCUPnet.jsp. Accessed 5 Jan 2010

16. Buchner AM, Sonnenberg A (2002) Epidemiology of Clostridium difficile infection in a large population of hospitalized U.S. military veterans. Dig Dis Sci 47:201-207

17. Adolor Corporation. Entereg ${ }^{\circledR}$ (alvimopan) Capsules. Prescribing Information. http://www.entereg.com/pdf/prescribing-information. pdf. Accessed 5 Jan 2010

18. Delaney CP, Weese JL, Hyman NH et al (2005) Phase III trial of alvimopan, a novel, peripherally acting, mu opioid antagonist, for postoperative ileus after major abdominal surgery. Dis Colon Rectum 48:1114-1129

19. Viscusi ER, Goldstein S, Witkowski T et al (2006) Alvimopan, a peripherally acting mu-opioid receptor antagonist, compared with placebo in postoperative ileus after major abdominal surgery: results of a randomized, double-blind, controlled study. Surg Endosc 20:64-70

20. Wolff BG, Michelassi F, Gerkin TM et al (2004) Alvimopan, a novel, peripherally acting mu opioid antagonist: results of a multicenter, randomized, double-blind, placebo-controlled, phase III trial of major abdominal surgery and postoperative ileus. Ann Surg 240:728-735

21. Ludwig KA, Enker WE, Delaney CP et al (2008) Gastrointestinal recovery in patients undergoing bowel resection: results of a randomized trial of alvimopan and placebo with a standardized accelerated postoperative care pathway. Arch Surg 143:10981105

22. Adolor Corporation. Entereg ${ }^{\circledR}$ (alvimopan) Capsules for postoperative ileus FDA Advisory Panel briefing document. http:// www.fda.gov/ohrms/DOCKETS/ac/08/briefing/2008-4336b1-02Adolor.pdf. Accessed 5 Jan 2010

23. Baum ML, Anish DS, Chalmers TC et al (1981) A survey of clinical trials of antibiotic prophylaxis in colon surgery: evidence against further use of no-treatment controls. N Engl J Med 305:795-799

24. Anderson DR, O’Brien BJ, Levine MN et al (1993) Efficacy and cost of low-molecular-weight heparin compared with standard heparin for the prevention of deep vein thrombosis after total hip arthroplasty. Ann Intern Med 119:1105-1112

25. Song F, Glenny AM (1998) Antimicrobial prophylaxis in colorectal surgery: a systematic review of randomised controlled trials. Health Technol Assess 2:1-110

26. Wells PS, Lensing AW, Hirsh J (1994) Graduated compression stockings in the prevention of postoperative venous thromboembolism. A meta-analysis. Arch Intern Med 154:67-72

27. Asgeirsson T, El-Badawi KI, Mahmood A et al (2010) Postoperative ileus: it costs more than you expect. J Am Coll Surg 210:228-231

28. Holte K, Kehlet H (2002) Postoperative ileus: progress towards effective management. Drugs 62:2603-2615

29. Schwandner O, Schiedeck TH, Bruch HP (1999) Advanced ageindication or contraindication for laparoscopic colorectal surgery? Dis Colon Rectum 42:356-362

30. Spivak H, Maele DV, Friedman I et al (1996) Colorectal surgery in octogenarians. J Am Coll Surg 183:46-50

31. Tartter PI (1988) Determinants of postoperative stay in patients with colorectal cancer. Implications for diagnostic-related groups. Dis Colon Rectum 31:694-698

32. Udelnow A, Leinung S, Schreiter D et al (2005) Impact of age on in-hospital mortality of surgical patients in a German university hospital. Arch Gerontol Geriatr 41:281-288 